\title{
Situación de salud de la población privada de la libertad en Colombia. Una revisión sistemática de la literatura*
}

\author{
Health Situation of Population Deprived of Liberty \\ in Colombia. A Systematic Review of the Literature
}

\section{Situação de saúde da população privada na Colômbia. Uma revisão sistemática da literatura}

\author{
Recepción: 06 de mayo de 2019. Aceptación: 28 deoctubre de 2019. \\ Publicación: 30 de junio de 2020. \\ DOI: https://doi.org/10.11144/Javeriana.rgps19.sspp \\ Mónica María Lopera Medina ${ }^{a}$ \\ Universidad de Antioquia, Colombia \\ ORCID: https://orcid.org/0000-0003-0144-057X \\ Jeniffer Hernández Pacheco \\ Universidad de Antioquia, Colombia \\ ORCID: https://orcid.org/0000-0002-5447-3714
}

\begin{abstract}
Cómo citar este artículo: Lopera Medina MM, Hernández Pacheco J. Situación de salud de la población privada de la libertad en Colombia. Una revisión sistemática de la literatura. Revista Gerencia y Políticas de Salud. 2020;19. https://doi.org/10.11144/Javeriana.rgps19.sspp
\end{abstract}

a Autora de correspondencia. Correo electrónico: monica.lopera@udea.edu.co 


\section{Resumen}

Las personas privadas de la libertad (PPL) están sujetas a condiciones adversas para su salud y problemas estructurales en los diferentes establecimientos penitenciarios y carcelarios (EPC) que restringen su resolución. Objetivo: analizar la evidencia científica que permita comprender la situación de salud de las PPL del país. Método: se realizó una revisión sistemática de las investigaciones en salud realizadas en las prisiones de Colombia, en algunas bases de datos y literatura gris, con los siguientes términos: "prisioneros"/"prisiones", "salud", "situación de salud", "condiciones de vida", "problemas de salud". Se abordan temas relacionados con la infraestructura, los principales problemas de salud (par población general y especial), así como sus determinantes cuando fueron explicados. Resultados: existe muy poca literatura científica que permita entender la situación de salud de las PPL. La mayoría de los estudios fueron referidos a problemas de salud mental y enfermedades infecciosas (TB e ITS). Los informes oficiales mostraron que las PPL experimentan condiciones desfavorables que limitan su salud. Conclusiones: en el país la escasa investigación científica limita la evidencia requerida para implementar políticas públicas apropiadas para mejorar la situación de salud, pero la información oficial no parece haber promovido un impacto positivo en la salud.

Palabras clave: prisioneros, prisiones, diagnóstico de la situación de salud, salud, contingencia carcelaria, derechos humanos, derecho a la salud.

\section{Resumo}

O pessoal privado da liberdade (PPL) está sujeito a condições adversas para sua saúde e problemas estruturais nos diferentes estabelecimentos penitenciários e carcerários (EPC) que restringem sua resolução. Objetivo: analisar a evidência científica que permita compreender a situação de saúde do PPL do país. Método: foi realizada revisão sistemática da pesquisa em saúde feita nas prisões da Colômbia, em algumas bases de dados e literatura cinzenta, com os seguintes termos: "prisioneiros"/“prisões", "saúde", "situação de saúde", "condições de vida", "problemas de saúde". Tópicos relacionados com a infraestrutura, os principais problemas de saúde (para população geral e especial) são abordados, mesmo como as determinantes, quando explicadas. Resultados: há muito pouca literatura científica que permita entender a situação de saúde do PPL. A maioria dos estudos foram referidos a problemas de saúde mental e doenças infeciosas (TB e ITS). Os relatórios oficiais mostraram que o PPL experimenta condições desfavoráveis que limitam a sua saúde. Conclusões: no país, escassas pesquisas científica limitam a evidência necessaria para implementar políticas públicas apropriadas para melhorar a situação de saúde, mas a informação oficial não parece promover um impacto positivo na saúde.

Palavras-chave: prisioneiros, prisões, diagnóstico da situação de saúde, saúde, contingência carcerária, direitos humanos, direito à saúde.

\section{Abstract}

People deprived of liberty (PDL) are subject to adverse conditions to the health and structural problems in the different prisons and penitentiary institutions (PPI). This make difficult to find a solution. Objective: To analyze the scientific evidence required to understand the health situation among the IP in our country. Method: A systematic review of health research carried out in the Colombian prisons was developed using some databases and grey literature compilations based on the following keywords: "prisoners"/"prisons", "health", "health situation", "life conditions", "health problems". This work addressed topics concerning the infrastructure, the main health problems (both in general and special populations), and the determining factors wherever described. Results: Scientific literature allowing understanding the health situation of PDL. Most of the studies were related to mental health problems and infectious disease (TB and STDs). Official reports showed that the PDL underwent unfavorable conditions that compromise their health. Conclusions: In our country the scant scientific research limits the evidence required to implement public policies appropriate to improve the IP health situation. On the other hand, the official reports have not meant a positive impact on their health.

Keywords: prisoners, prisons, health situation diagnosis, health, prison contingency, Human Rights, right to health. 


\section{Introducción}

La protección de los derechos humanos, y en especial el derecho a la salud de los prisioneros, en adelante referidos como personas privadas de la libertad - PPL_, es una obligación del Estado, independiente del tiempo o la situación judicial del individuo. El Estado debe garantizar las acciones individuales y colectivas que permitan mantener la salud, prevenir la enfermedad, tratar eficaz y oportunamente las enfermedades que padezcan los individuos en prisión y mejorar la calidad de vida (1).

\section{Protección de los derechos humanos de las PPL}

Los derechos de las PPL están orientados por los estándares mínimos de cumplimiento adoptados por la Asamblea general de la Organización de las Naciones Unidas en su Resolución 45/111 del 14 de diciembre de 1990 (2). En el país, dichos estándares están protegidos por la Constitución Política, las garantías contempladas en el código penitenciario (Ley 65 de 1993 y Ley 1709 de 2014, que la modifica) (3, 4), así como las sentencias T-388 de 2013 y T-762 de $2015(5,6,7)$.

El estándar constitucional mínimo que debe cumplir una política criminal respetuosa de los derechos humanos de las PPL se aboca a la garantía del derecho a la alimentación, el derecho a la salud, el derecho a la salubridad e higiene, la disponibilidad de servicios de salud, el derecho a no vivir en condiciones de hacinamiento, el derecho a la separación entre sindicados y condenados, y el derecho a la resocialización (8).

\section{La situación de salud y los determinantes sociales de la salud}

La situación de salud que hoy viven las PPL trasciende el fenómeno biomédico de saludenfermedad. Un abordaje más integral permite entenderla como resultante de la interacción entre los determinantes del orden estructural, institucional y personal (9). La situación de salud de las PPL es un problema de salud pública susceptible de transformación prioritaria.

Las estructuras de segregación social, las deficientes condiciones de vida asociadas con la pobreza, la marginación y la exclusión de la población antes de la detención, sumadas a una política criminal punitiva, favorecen el hacinamiento en los EPC y sus importantes consecuencias. Por otra parte, la asignación presupuestal y las prioridades políticas influyen en el estado actual de la infraestructura carcelaria, situación que determina las condiciones de salubridad precarias, con aumento de enfermedades infecciosas, problemas de convivencia y salud mental, entre otros (10).

Desde una perspectiva de salud pública, la compleja situación de salud en los EPC representa un riesgo no solo para las PPL, sino también para sus familias y el cuerpo de vigilancia y custodia y para la salud pública, por lo tanto, para lograr una intervención efectiva en los Establecimientos Penitenciarios y Carcelarios, EPC e impactar en los determinantes sociales de la salud deben 
implementarse políticas penales y carcelarias que atiendan las necesidades en salud, así como los factores ligados a las condiciones propias del confinamiento (11).

\section{Características estructurales de los EPC}

El sistema de prisiones en Colombia fue creado en 1914 mediante la Ley 35 (12). Actualmente, el Sistema Penitenciario y Carcelario está integrado por el Instituto Nacional Penitenciario y Carcelario, Inpec, adscrito al Ministerio de Justicia y del Derecho, los centros de reclusión, la Escuela Penitenciaria Nacional, la Unidad de Servicios Penitenciarios, USPEC, y los recientemente adicionados; el Ministerio de la Protección Social y el Instituto Colombiano de Bienestar Familiar (Ley 1709 - 2014) (4). El Inpec está organizado en un nivel central, ubicado en Bogotá, y seis Direcciones Regionales que coordinan, administran y vigilan los 136 EPC, también llamados Establecimientos de Reclusión de Orden Nacional, ERON (13, 14). Estos se clasifican en cárceles y penitenciarías, de acuerdo con el tipo de población privada de la libertad. Las cárceles son establecimientos de detención preventiva, prevista para retención y vigilancia de sindicados, es decir, personas que están pendientes de resolver su proceso jurídico, mientras las penitenciarías son establecimientos destinados para condenados (3). Las características estructurales de los EPC constituyen determinantes de la situación de salud de los PPL.

La situación de salud de las PPL ha sido develada principalmente por las entidades de control y las fuentes periodísticas, sin embargo, hasta el momento no existe información científica sistemática que permita conocer la situación de las PPL en el país. La identificación de esta situación es condición necesaria para efectuar políticas públicas dirigidas al mejoramiento de la salud de las PPL y de la comunidad.

\section{Método}

Se realizó una revisión sistemática de la literatura, de acuerdo con las orientaciones aplicables sugeridos por Prisma, Prefered Reporting Items for systematic review and Meta-analysis (15).

\section{Criterios de elegibilidad}

La revisión se dirigió a la búsqueda de estudios, bases de datos e informes oficiales publicados entre 2000-2018 que informaran la situación de salud de las PPL de Colombia.

\section{Fuentes y búsqueda de información}

La búsqueda de información se realizó usando una estrategia de pregunta PICO adaptada a intereses y necesidades en salud pública: población, situación de interés (problemas y necesidades en salud), resultado y lugar (PPRL). 
La búsqueda de la situación de salud se efectuó en las bases de datos SciELO, PubMed, Science Direct y Embase, utilizando los siguientes términos "Prisioneros"/“Prisiones", "salud", "situación de salud", "condiciones de vida", "problemas de salud", así como sus sinónimos correspondientes. La búsqueda se efectuó en inglés y español. La literatura gris se buscó en las páginas oficiales del Inpec, la autoridad sanitaria y entes de control del Estado, infografías periodísticas, así como trabajos de grado sugeridos por expertos en el tema. Se incluyeron solo estudios con texto completo. La búsqueda se realizó durante el 2018.

\section{Selección de los estudios}

Se incluyeron todos los estudios, bases de datos e informes que cumplieron los criterios de búsqueda y estuvieron disponibles en texto completo. Se excluyeron los artículos que hacían referencia a otro país, aquellos cuyo objeto difería del propósito de esta revisión o, artículos que presentaran datos agregados, y por lo tanto impidieran diferenciar los datos del país. No se incluyó información referente a la atención porque será abordada en otro estudio.

\section{Procesamiento de la información}

Una vez generada la estrategia de búsqueda, que fue ejecutada de forma independiente por dos miembros del equipo de trabajo, se verificó que los documentos y bases de datos descargados estuvieran exentos de duplicidad. Para realizar la categorización se realizó una primera depuración con temas emergentes. La base de datos oficial del Inpec, que contenía información de las condiciones sociodemográficas de las PPL, fue analizada mediante estadísticas descriptivas.

\section{Resultados}

En la búsqueda de bibliografía se identificaron 82 artículos, tanto de literatura científica como de literatura gris. De estos se descartaron 6 duplicados y se excluyeron 28, porque hacían referencia a otro país, diferían del propósito del artículo o tenían datos indiferenciables. En total se revisaron 16 artículos académicos publicados en revistas indexadas y 15 de la literatura gris. La totalidad de los estudios indexados eran observacionales o cualitativos. En las páginas oficiales se encontró una base de datos con información cuantitativa consignada en Excel; con las condiciones sociodemográficas de las PPL (Ver Figura 1). 


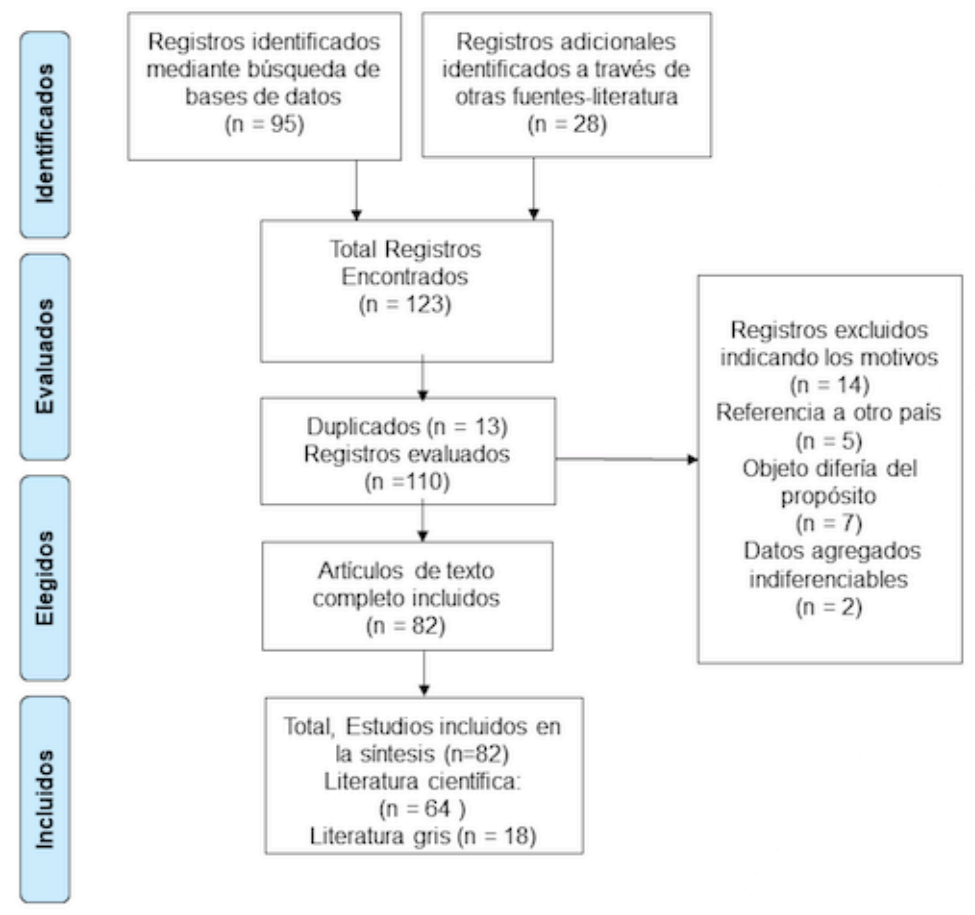

Figura 1. Prefered Reporting Items for systematic review and Meta-analysis, Prisma. Situación de salud de las personas privadas de libertad en Colombia elaboración propia.

A continuación, se describen los elementos de infraestructura, características sociodemográficas de las PPL, condiciones de salud como salud mental, nutrición, enfermedades infecciosas, enfermedades crónicas no transmisibles y problemas de salud de las poblaciones con necesidades especiales. En esta última categoría se incluyeron adultos mayores, mujeres y sus niños, así como personas con diversidad de género.

\section{Características estructurales de los EPC como determinante de los procesos de salud}

Los establecimientos penitenciarios y carcelarios (EPC) sufren importantes problemas estructurales que afectan la salud de las PPL, en tanto que las construcciones presentan un índice de deterioro y envejecimiento superior al 50\%, lo que representa limitaciones en espacios comunes, áreas educativas y dormitorios, entre otros. Las edificaciones en las que operan los EPC se clasifican como de primera, segunda y tercera generación, de acuerdo con la fecha de construcción y las condiciones de infraestructura $(13,16)$ (Ver Tabla 1). Del total de los EPC, solo los establecimientos construidos después de 2010 (tercera generación) cumplen con la norma Colombiana de Diseño y Construcción Sismo Resistente NSR98 (17). 
Tabla 1. Clasificación de los EPC según características estructurales y de antigüedad

\begin{tabular}{|c|c|c|c|c|}
\hline Clasificación & Cantidad & Antigüedad & Caracteristicas & $\begin{array}{c}\% \text { de PPL } \\
\text { que albergan }\end{array}$ \\
\hline $\begin{array}{c}\text { Primera } \\
\text { generación }\end{array}$ & 121 & $\begin{array}{c}\text { Entre } 11 \text { y } \\
400 \text { años }\end{array}$ & $\begin{array}{l}\text { Deterioro en muros, redes eléctricas y } \\
\text { alumbrado, fallas en redes } \\
\text { hidrosanitarias, en plantas de tratamiento } \\
\text { de agua potable, problemas en los } \\
\text { alojamientos y la cocina. Problemas de } \\
\text { inundaciones. Infraestructura para } \\
\text { servicios de salud carente de garantias } \\
\text { para su funcionamiento. }\end{array}$ & $62 \%$ \\
\hline $\begin{array}{c}\text { Segunda } \\
\text { generación }\end{array}$ & 6 & $\begin{array}{c}\text { Entre } 3 \text { y } 10 \\
\text { años }\end{array}$ & $\begin{array}{l}\text { Se construyeron a finales de la década de } \\
1990 \text { y principios del } 2000 \text {. Cuentan con } \\
\text { mejores condiciones de operación y } \\
\text { funcionamiento, pero persisten los } \\
\text { problemas de infraestructura y } \\
\text { operación, generados principalmente por } \\
\text { la falta de mantenimiento preventivo y } \\
\text { correctivo. }\end{array}$ & $11 \%$ \\
\hline $\begin{array}{c}\text { Tercera } \\
\text { generación }\end{array}$ & 10 & $\begin{array}{c}\text { Entre } 1 \text { y } 3 \\
\text { años }\end{array}$ & $\begin{array}{l}\text { Son construcciones de la última década y } \\
\text { cumplen con las caracteristicas técnicas } \\
\text { requeridas. }\end{array}$ & $27 \%$ \\
\hline
\end{tabular}

Fuente: elaboración propia, basada en fuentes secundarias $(18,19)$.

Desde larga data, los organismos oficiales han descrito importantes fallas en la estructura carcelaria, tales como carencia de zonas perimetrales de seguridad, limitaciones de tamaño, deficiencias en las condiciones de infraestructura, hacinamiento, problemas con el suministro de agua, iluminación y ventilación, así como deficiente infraestructura para la atención médica y saturación en la atención sanitaria (20) y, en especial, en la infraestructura de los establecimientos con vocación médico-asistencial, requeridos para la atención de las PPL que padecen enfermedades mentales graves, aquellos inimputables o con trastornos mentales sobrevinientes a la condena y contemplada en el marco jurídico del país $(4,21)$. Estas características son importantes, toda vez que las condiciones en materia constructiva, tamaño y adecuación de espacios determinan las condiciones de vida, higiene y salubridad que afectan negativamente la salud de las PPL, sus familias y los funcionarios que allí laboran (20).

Particularmente en Colombia, se presenta una situación de sobrepoblación que, sumada a otros factores de tipo medioambientales como la precaria eliminación de desechos, la dificultad en acceso a programas de atención y hechos de violencia, entre otros, propicia la presencia enfermedades físicas y mentales que afectan continuamente las condiciones de salud de esta población (16). 


\section{Características de la Población Privada de la Libertad}

Hasta enero de 2017, el número de PPL era de 118.925. Este número representa el 1\% sobre el total de PPL en el mundo. El país ocupa el puesto trece en el mundo con mayor número de PPL (22). La tasa de crecimiento anual de PPL en el país es de aproximadamente 14\%. Este crecimiento puede ser atribuible tanto a las desigualdades sociales, como a causas relacionadas con el congestionamiento del sistema penitenciario, que mantiene al $32 \%$ de ellos como sindicados $(23,24)$.

Para 2017, el 6,5\% (7800) de las PPL eran mujeres y el 93,4\% hombres (111.125) (25). El 72\% (85.499) se encontraban en edades productivas de 25 a 49 años (26). Para 2017 vivían en los EPC del país 108 hijos de mujeres privadas de la libertad (27). En 2017, el 8,3\% de las PPL tenía alguna condición especial referida a la edad (población adulta mayor), condición biomédica, étnica o país de origen (26).

Un estudio realizado en un EPC de Medellín reportó que un gran porcentaje de las PPL pertenece a los grupos más vulnerables de la sociedad, tienen escasos recursos, bajo nivel educativo y condiciones laborales desfavorables antes de la detención. Según este estudio, el 82,6\% de la población pertenecía a los estratos 0-3 y el 69\% tenía nivel educativo de bachillerato incompleto o inferior (28). Esta situación no parece transformarse de manera importante durante su reclusión, puesto que las oportunidades de trabajo, estudio y uso de tiempo libre son limitadas (29). Al momento del reporte, la empresa privada tenía, 61 maquilas en los penales, para emplear a 1441 PPL (1,2\%), cuyos ingresos estaban por debajo del salario mínimo legal (27).

No se hallaron cifras nacionales de la cobertura en salud de las PPL, sin embargo, un estudio realizado en el año 2014 en un EPC de Medellín señaló que el 90,8\% contaba con aseguramiento a una EPS con relación contractual con el Inpec. El 9,2\% restante pertenecía al Régimen Contributivo, RC, o era Pobre No Asegurado, PNA (28). Hay que aclarar que, bajo la actual organización del sistema de salud en las cárceles, todas aquellas PPL que no están afiliadas al Régimen Contributivo son atendidas por entidades contratadas por la Unidad de Servicios Penitenciarios y Carcelarios, USPEC. Las características sociodemográficas de las PPL en Colombia se pueden observar en Tabla 2. 
Tabla 2. Características sociodemográficas de las personas privadas de la libertad del país. Enero de 2017

\begin{tabular}{|c|c|c|c|}
\hline Caracteristica & & $\begin{array}{l}\text { N. } \\
(118.925)\end{array}$ & $\begin{array}{l}\text { Porcentaje } \\
\%\end{array}$ \\
\hline \multirow{2}{*}{ Sexo } & Hombre & 111.125 & $93,44 \%$ \\
\hline & Mujer & 7800 & $6,55 \%$ \\
\hline \multirow{10}{*}{ Nivel académico al ingresar al programa } & lletrado & 6398 & $5,37 \%$ \\
\hline & Estudiante & 42.950 & $36.12 \%$ \\
\hline & Básica Primaria & 72.950 & 30,1276 \\
\hline & Estudiante & & \\
\hline & Básica & 65.703 & $55,24 \%$ \\
\hline & Secundaria & & \\
\hline & Técnico & 1721 & $1,45 \%$ \\
\hline & Tecnológico & 556 & $0,48 \%$ \\
\hline & $\begin{array}{l}\text { Profesional } \\
\text { completo }\end{array}$ & 1358 & $1,14 \%$ \\
\hline & Especializado & 239 & $0,2 \%$ \\
\hline \multirow{8}{*}{ Población Según grupos de población } & Indigena & 1238 & $1,04 \%$ \\
\hline & Afrocolombiano & 4038 & $3,4 \%$ \\
\hline & Extranjero & 833 & $0,7 \%$ \\
\hline & Adulto mayor & 2548 & $2,14 \%$ \\
\hline & Madre Lactaste & 14 & $0,01 \%$ \\
\hline & Madre Gicstante & 68 & $0,06 \%$ \\
\hline & Discapacitados & 1086 & $0,91 \%$ \\
\hline & Imputables & $\$ 1$ & $0,04 \%$ \\
\hline \multirow{2}{*}{ Sindicades } & Hombres & 35.655 & $92,96 \%$ \\
\hline & Mujeres & 2701 & $7,04 \%$ \\
\hline \multirow{2}{*}{ Condenados } & Hombres & 75.470 & $93,67 \%$ \\
\hline & Mujeres & 5099 & $6,33 \%$ \\
\hline \multirow{2}{*}{$\begin{array}{l}\text { Poblacion de internos octupados en trabajo, } \\
\text { estudio y ensenianza (enero de } 2017 \text { ) }\end{array}$} & Hombres & 88.144 & $7,9 \%$ \\
\hline & Mujeres & 6457 & $82 \%$ \\
\hline
\end{tabular}

Fuente: elaboración propia, basada en fuentes secundarias $(18,19)$.

\section{Condiciones de salud y sus determinantes}

Hasta el momento, en Colombia existe muy poca información científica que ofrezca un panorama claro de la situación de salud de las PPL. La Organización de las Naciones Unidas (ONU) ha afirmado que muchas de ellas ingresan a los centros de reclusión con problemas de salud (18). Otras fuentes oficiales del país han revelado las precarias condiciones de salud en los penales $(30,31,32)$. Entre los principales problemas que experimentan las PPL son el hacinamiento, los problemas de salud mental, los problemas nutricionales, las enfermedades crónicas no transmisibles y otros problemas referidos a poblaciones con necesidades especiales, como los adultos mayores, las mujeres privadas de la libertad y sus hijos, así como la población sexualmente diversa. Estos problemas de salud se describen a continuación y se sintetizan en las Tablas 3 y 4. 


\section{Hacinamiento}

En Colombia, el número de PPL supera ampliamente la capacidad poblacional de los EPC, estimado para 78.418. Hasta el 2017 los EPC albergan a 118.925 personas, esto representa un hacinamiento del $51,7 \%$. Este fenómeno no se comporta de manera similar en las seis regionales; este oscila entre el 59\% y 76\%, donde la Regional norte es la más afectada (26). Algunos estudios han develado las dificultades de hacinamiento y problemas de salubridad de los EPC $(19,33)$. Según Álvarez y Cadena, el hacinamiento se ha duplicado entre 2008 y 2017, donde pasó de $25 \%$ al $51,2 \%(31)$.

Con base en las deficientes condiciones de los EPC, para el año 2012 se cerraron 26 establecimientos que no cumplían con las condiciones adecuadas para la garantía de los derechos humanos, situación que llevo a declarar la emergencia carcelaria (34). Un análisis detallado del hacinamiento por regiones se puede observar en la Tabla 3.

Tabla 3. PPL por regional y el porcentaje de hacinamiento respectivo. Colombia, Año 2017

\begin{tabular}{lccccc}
\hline \multirow{2}{*}{ Regional } & Capacidad & Total & \multicolumn{2}{c}{ Sexo } & \\
\cline { 4 - 5 } & & población & $\mathrm{H}$ & $\mathrm{M}$ & \\
Regional central & 29.393 & 40.530 & 38.063 & 2467 & $37,9 \%$ \\
Regional occidente & 14.663 & 24.101 & 22.400 & 1701 & $64,4 \%$ \\
Regional norte & 7735 & 13.642 & 13.259 & 383 & $76,4 \%$ \\
Regional oriente & 7575 & 12.163 & 11.410 & 753 & $60,6 \%$ \\
Regional noroeste & 8485 & 14.816 & 13.444 & 1372 & $74,6 \%$ \\
Regional viejo caldas & 10.567 & 13.673 & 12.549 & 1124 & $29,4 \%$ \\
\multicolumn{1}{c}{ Total } & 78.418 & 118.925 & 111.125 & 7800 & $51,7 \%$ \\
\hline
\end{tabular}

Fuente: tomado y Modificado del Instituto Nacional Penitenciario y Carcelario. Estadísticas 2017 (26).

\section{Problemas de salud mental}

Los problemas de salud mental se cuentan entre las principales causas de pérdida de salud de esta población. En los EPC son comunes los problemas de convivencia permanente, abusos de poder, violencia y consumo de Sustancias Psicoactivas, SPA, intento de suicidio y un alto número de personas con otras enfermedades mentales, EM. Se estima que la ocurrencia de trastornos psiquiátricos es siete veces mayor en las personas que se encuentran privadas de la libertad que en la población general, siendo en su mayoría trastornos del afecto $(35,36)$.

En un estudio realizado en un EPC de Manizales que valoró la situación de salud mental, según la Escala Goldberg General Health Questionnaire, se reportó que de las 39 en mujeres incluidas, el $33,3 \%$ mostró deterioro alto de la salud mental y el $22,2 \%$ deterioro bajo (37). Según el informe 
de la Defensoría del Pueblo, la violencia y el consumo SPA se incrementan cuando se conjugan las condiciones estructurales en las que viven estas personas y el poco reclamo que tienen las mismas para hacer valer sus derechos fundamentales (32). Con relación al consumo de SPA, un trabajo de investigación en un EPC de Medellín mostró los hábitos de consumo, encontrando que el 57,3\% fumaba cigarrillo, 49,8\% consumía de drogas fumadas como marihuana, 26\% drogas inhaladas como perico y cocaína, y 17,5\% consumía licor. Los resultados indicaron que, para algunas de estas sustancias, el consumo aumentó durante el tiempo de permanencia en los EPC (28). En el estudio de Jaramillo y colaboradores, en Manizales, se encontró que el consumo de SPA entre mujeres fue de 12,8\% (37), y el estudio de Uribe et al. encontró 74,1\% de consumo de sustancias psicoactivas en una EPC de Bucaramanga (38). El estudio de Riaño en un EPC de Putumayo reveló que 35,3\% de los encuestados consumía sustancias psicoactivas, y el 54,2\% de ellos manifestó que ya tenían un hábito de consumo desde antes de ingresar al EPC (33). Por otro lado, Gómez encontró que el consumo de SPA varió entre $2 \%$ y 47\%, dependiendo del EPC (39).

En Colombia, para el año 2017 existían 2884 personas con EM (24). Uribe et al. realizaron un estudio para identificar depresión y ansiedad en un EPC de Bucaramanga, mediante la aplicación del Inventario de Depresión Estado Rasgo (IDER) y el Inventario de Ansiedad Estado Rasgo (STAI), los autores encontraron que el 16,7\% calificó como estado de manifestaciones depresivas y un 43,68\% como rasgo, mientras que la afectación ansiosa se mostró en un $8,03 \%$ como estado y en un $85,7 \%$ como rasgo (38).

Por su parte, Jaramillo et al. identificaron orientación suicida en el 14,9\% de las PPL en una EPC de Medellín. Esta fue mayor en personas solteras, menores de 30 años, quienes habían tenido intento previo, y antecedentes de violencia intrafamiliar (40).

Mojica et al. realizaron un estudio en un EPC de país con el fin de describir el riesgo suicida y su relación con la desesperanzan y la depresión. Mediante la Escala de Riesgo Suicida de Plutchick, la Escala de Desesperanza de Beck y el Inventario de Depresión de Beck, los autores llegaron a la conclusión de que el 20\% de los participantes presentaba riesgo suicida, y que este estaba asociado significativamente cuando coexistían la desesperanza y la depresión, pero no a las variables sociodemográficas estudiadas (41). Por el contrario, Gómez y colaboradores encontraron que el riesgo de suicidio se asociaba con factores como una menor edad, dificultades para ajustarse a las normas del centro y con antecedentes de enfermedades físicas y de dificultades sexuales (39).

Álvarez y colaboradores describen los diagnósticos clínicos de los internos de la unidad de salud mental de la cárcel La Modelo y de la cárcel Villahermosa (31). Entre las EM más prevalentes en las PPL se presentaron, en su orden, la esquizofrenia, farmacodependencia, trastorno de ansiedad, trastorno depresivo y trastorno afectivo bipolar (31). Además de la complejidad clínica de estas enfermedades, las personas con EM son frecuentemente rechazadas y golpeadas por las demás PPL (27). Se ha notificado que, en algunos casos, las PPL con padecimientos psiquiátricos se ubican en áreas pabellones psiquiátricos, pero en condiciones de hacinamiento y 
sin supervisión continua de especialistas. Cuando no existen los espacios de aislamiento y deben convivir con el resto de los PPL, son maltratados y revulnerabilizados, lo que se convierte en un ambiente perjudicial para la salud e integridad de todas las PPL. Estas complejas enfermedades plantean retos de carácter logístico, sanitario y ético al sistema penitenciario y al sistema de salud (42).

Otros estudios han descrito los elementos emocionales predominantes. Así, en un estudio realizado en 13 EPC del país, se identificó que las emociones que alcanzaron puntajes más extremos fueron tristeza, soledad y tranquilidad (altas), junto a una baja alegría y confianza en la institución (43).

\section{Problemas nutricionales e inocuidad de los alimentos}

No solo se han realizado estudios relacionados con la nutrición, sino también con la inocuidad de los alimentos. Informes oficiales mostraron que en los EPC del país se presentan problemas asociados a la deficiencia en cantidad y calidad de los alimentos (29). Un estudio de mujeres privadas de la libertad reportó baja talla, pero no bajo peso. Así mismo, se encontró que el acceso a las dietas terapéuticas fue bajo (37). Bejarano et al. reportaron que durante el 2014 se habían notificado 20 brotes de Enfermedad Diarreica Aguda, EDA, como síntoma de las Enfermedades Transmitidas por Alimentos, ETA, así: $35 \%$ atribuida al servicio de alimentos, el $35 \%$ a los alimentos "sin control" sanitario ${ }^{1}$, el $18 \%$ al consumo de agua sin tratamiento adecuado y el $12 \%$ debido a casos indeterminados (44).

\section{Enfermedades infecciosas}

Las deficientes condiciones espaciales y sociales de los EPC exacerban el riesgo de desarrollo de enfermedades infectocontagiosas y, en especial, aquellas de transmisión sexual, tuberculosis (TB) y de contacto directo (45).

La enfermedad más estudiada en los EPC del país es la TB. Los estudios determinan la incidencia de TB pulmonar y extrapulmonar y altas tasas de conversión durante la permanencia en los penales. Específicamente en ciudades como Medellín y Bucaramanga (2010-2011) se encontró que la incidencia de TB fue 20 veces mayor en los centros de reclusión, que en la población general $(46,47)$.

Un estudio con 1014 PPL encontró un riesgo anual de infección tuberculosa entre 2,7\% y $5,09 \%$ en dos EPC (48). Otro estudio finalizado en 2012 encontró una incidencia anual de 505 casos/100.000 PPL en 4 penales del país, donde estaban recluidas 9507 PPL. Los factores de riesgo asociados son situaciones de encarcelamiento previo y el tiempo de reclusión, bajo peso, 
haber tenido historia previa de TB o contacto previo con un infectado, problemas de ventilación, pocos programas de prevención, entre otros (49).

Según Gómez, la prevalencia de coinfección TB/VIH en PPL entre 2010-2012 fue de 12\%. El 94\% de los pacientes no habían recibido ningún tratamiento para la tuberculosis (50). Cifra similar fue hallada en un estudio realizado en Calarcá en 2005, en el que se indicó que el $94,4 \%$ de los pacientes con diagnóstico de TB manifestaron no haber recibido tratamiento antituberculoso (51).

Contrario a lo esperado, se encontraron muy pocos estudios referidos al VIH y otras infecciones de transmisión Sexual, ITS. Sánchez reportó que el VIH/Sida afectaba a 611 PPL (28), y Gaviria reportó una prevalencia de $11 \%$ de VIH en penales de Barranquilla, e importantes factores de riesgo para la expansión de la infección (52). Se ha informado que las personas con VIH no acceden oportunamente a los medicamentos, lo que produce deterioro de su estado de salud. Frecuentemente sufren consecuencias sociales relacionadas con menores oportunidades laborales y de integración con otros internos (29). Un estudio realizado en las cárceles de Colombia concluyó que la infección por VIH genera gran morbilidad en el mundo y afecta especialmente a las personas privadas de la libertad, debido a la limitada prevención, los diagnósticos tardíos y el tratamiento inoportuno. En estos centros las normas de transmisión más frecuentes son por contacto sexual y uso de drogas intravenosas (53).

Se encontraron dos estudios referidos a hepatitis B $(54,55)$. En el estudio de De la Hoz, realizado en un EPC del Tolima, se encontró una alta prevalencia de Hepatitis B de 34\%, siendo la conducta de riesgo sexual el factor principal para la transmisión (54). Por su parte, Castañeda, en una muestra de 16 sueros de PPL, encontró una prevalencia de infección aguda determinada por AntiHBc/IgM de 31,3\%. De los 213 encuestados, se evidenció un bajo uso del preservativo (25\%), alta prevalencia autorreportada de ITS, siendo las más comunes el VIH $(40,4 \%)$, la gonorrea $(23,9 \%)$ y la sífilis $(15,5 \%)$. Este estudio también señaló el desconocimiento sobre la hepatitis y la presencia de otros factores de riesgo asociados como uso de máquinas rasuradoras y carencia de autoclave para la esterilización de equipos odontológicos (55). Similar al anterior, un estudio en un EPC de Putumayo encontró que, aunque el 90\% manifestaba conocer las ITS, el 25,7\% respondió de manera errónea a conocimientos específicos, y 19,8\% no respondió (30).

Otras enfermedades infecciosas de interés en Salud Pública reportadas en los EPC fueron leishmaniosis cutánea, leptospirosis, dengue, infección respiratoria aguda (IRA) y parotiditis $(28,54,55,56)$.

\section{Enfermedades crónicas no transmisibles}

Con respecto la enfermedad crónica, un estudio en un EPC de Pereira, que evaluó 61 hombres con edad promedio de 48 años, mostró una prevalencia de $11 \%$ de enfermedad cardiovascular, ECV establecida. Los factores de riesgo más prevalentes para el padecimiento de estas 
enfermedades fueron la poca ingesta de vegetales (70\%), obesidad central (66\%), sedentarismo $(62 \%)$ y tabaquismo (54\%) (57).

\section{Población con necesidades especiales}

Con respecto a los problemas de salud de las poblaciones con necesidades especiales, esta revisión identificó los siguientes grupos: adultos mayores, mujeres privadas de la libertad y personas con diversidad de género.

\section{Adultos mayores}

Para el 2017, los EPC del país albergaban 2548 (2,14\%) adultos mayores (25). Estas personas requieren de cuidados especiales que no pueden ser garantizados en los EPC, y bajo estas circunstancias están propensos a problemas de salud. En 2013, se analizaron las condiciones de vida de adultos mayores en algunos EPC del país y se evidenció el incumplimiento de reglas internacionales de derechos humanos aplicables a esta población en lo relacionado con infraestructura y dotación para la atención básica. Este asunto finalmente influye en la percepción negativa del estado de la salud de estas personas (58).

Un estudio de la Universidad del Rosario mostró que los adultos mayores tenían disminución de las capacidades físicas, enfermedades cardiovasculares, virales, bacterianas, alérgicas, respiratorias, reumatológicas, del sistema óseo, pérdida progresiva de la visión y del oído, cáncer o fallas renales. Entre los problemas de salud mental se reportó el estigma debido a su edad, el estrés, la depresión, malestares psicopatológicos y problemas de memoria. La investigación concluyó que tener la condición de adulto mayor en los EPC implica dependencia y desprotección, y por tanto señala la necesidad de cuidados especiales (59).

Finalmente, si bien el Inpec diseñó un programa de integración social para grupos con condiciones excepcionales, no es clara una política de tratamiento penitenciario diferencial para esta población (58).

\section{Mujeres privadas de la libertad y sus hijos}

El 6,6\% (7800) de las PPL son mujeres. Además de padecer enfermedades similares a las que padece la población masculina; las mujeres presentan necesidades específicas relacionadas con afecciones ginecológicas, salud sexual y reproductiva y problemas de salud mental $(60,61,62)$.

Un estudio realizado en 2018 indicó que un porcentaje importante de mujeres privadas de la libertad manifestó condiciones precarias de vida en prisión (sin cama, presencia de plagas en dormitorios) y dificultades de acceso a los servicios de salud. Así mismo, describe las 
consecuencias diferenciales de la prisionalización en los niños, tanto cuando la madre ingresa al EPC o cuando nace dentro de él. Algunas consecuencias para los niños que nacen en los EPC están referidas principalmente a problemas de habla y motricidad, mientras aquellos que ingresan con posterioridad manifiestan problemas de control de esfínteres, dificultades del habla, elementos emocionales como la impulsividad, la agresividad, dificultades con el sueño, déficit de atención, hiperactividad, estrés, problemas con la alimentación. Ambos presentan problemas de adaptación a espacios abiertos (61).

Un estudio de tipo transversal realizado a mujeres que se encontraban recluidas en un EPC de Bucaramanga con edades entre los 19 y 49 años, mediante el cual se pretendía visibilizar la frecuencia de infecciones vaginales en las mujeres recluidas, evidenció baja frecuencia en el uso de preservativos. También, se logró identificar que las mujeres con antecedentes de consumo de tabaco tuvieron un riesgo de 1,71 veces de contraer una infección vaginal frente aquellas que no consumían tabaco. Igualmente, las mujeres con edad superior a los 35 años presentaron un riesgo de 1,4 veces de contraer una infección vaginal frente a las que tenían edades inferiores (63).

Los estudios muestran que entre los problemas de salud mental más significativos que sufren las mujeres recluidas en los EPC se encuentran los trastornos depresivos, de ansiedad con ataques de pánico, estrés postraumático y drogodependencias, tal es el caso de un estudio de investigación-acción realizado en una EPC de Barranquilla, en el que se encontró que de las 130 mujeres atendidas, el 50\% presentaba trastornos depresivos de considerable gravedad, el $16,6 \%$ depresión, pensamientos asociados a la muerte e intentos de suicidio. Mayores niveles de estrés se producen en el proceso de crianza de los hijos, independiente de si conviven o no con ellos en los EPC (64).

Poco se sabe sobre experiencias en la salud de los niños convivientes con sus madres en los EPC (65), pero se han informado problemas nutricionales y atraso en su desarrollo (61). En un estudio realizado para una EPC de Bogotá, se identificó que el 86\% de las internas tienen en promedio entre 1 y 2 hijos. De estas, un 5\% convivían con ellos en la EPC, sin las condiciones requeridas. Como consecuencia, se producen efectos negativos, tanto para la madre como para los hijos. Estos últimos quedan desprotegidos, con dificultades para el sustento económico, lo que los puede llevar a trabajar desde edades muy tempranas (66). Finalmente, se registró que cuando las madres son separadas de sus hijos al cumplir los tres años, no cuentan procesos de asesoría psicológica para sobrellevar el momento de la separación. En este aspecto, resulta fundamental el seguimiento profesional para afrontar este momento (60).

\section{Personas con diversidad de género}

En Colombia se relatan las difíciles condiciones que enfrentan estas personas en los EPC, relacionadas con maltrato físico y psicológico, ITS, drogodependencias y principalmente las complicaciones derivadas de procedimientos de transformación corporal, interrumpidos una vez se ingresa a un EPC (67). Parra describió las características de la población sexualmente diversa en los EPC del país, reportando que, según el Sistema de Información de Sistematización 
Integral del Sistema Penitenciario y Carcelario, Sisipec, a 2013 existían 815 personas de la comunidad LGTBI en el país, y reconoció el estado especial de vulnerabilidad de esta población en los EPC (68).

Un análisis documental de las leyes penitenciarias del país, y en especial la ley 1709 de 2014, concluye que la normatividad avanza hacia las mujeres, pero no asume una política integral de género que dé respuestas especializadas desde enfoques diferenciales y alternativos, especialmente para las madres y sus infantes (60).

\section{Otros problemas de salud}

De acuerdo con varios estudios realizados en EPC, de Medellín, otras condiciones mórbidas prevalentes son: enfermedades respiratorias, osteomusculares y neurológicas (69). Riaño describió también la prevalencia de la morbilidad general en un EPC de Putumayo (33).

Tabla 4. Situación de salud: Problemas o necesidades en salud

\begin{tabular}{|c|c|c|c|}
\hline \multicolumn{2}{|c|}{ Situacion de salud problemas o nocesidades en salud } & Publicactones cientificas & Literatura gris \\
\hline $\begin{array}{l}\text { Infraestructura y } \\
\text { caracteristicas } \\
\text { contextuales }\end{array}$ & $\begin{array}{l}\text { Los establecimientos no curiplen con normas de disctio y } \\
\text { construcción. } \\
\text { Los espacios en los establecimientos son reducidos. } \\
\text { Problemas en suministro de agua potable. } \\
\text { Problemas de lluminación y vestilacion. } \\
\text { Sobrepoblación que excede la capacidad poblacional. }\end{array}$ & (31) & $(13,15,17,20,32)$ \\
\hline $\begin{array}{l}\text { Conidiciones } \\
\text { sociodemográficas y } \\
\text { de aseguramiento }\end{array}$ & $\begin{array}{l}\text { Afiliación al Régimen Sobsidiado (1 de mayo de 2017): } \\
7493 \text { PPL (6,5\%) } \\
\text { Alta presencia de grupos vulnerables (condición estructural } \\
\text { de bajo nivel socioeconóenico, educativo y laboral). }\end{array}$ & (28) & $(22-27)$ \\
\hline $\begin{array}{l}\text { Situacion de salud } \\
\text { (datos generales) }\end{array}$ & $\begin{array}{l}\text { Situacion de salud precaria al ingreso. } \\
\text { Situación de salud en prisión. } \\
\text { Consecuencias asociadas al hacinamiento y las condiciones } \\
\text { de infraestroctura. } \\
\text { Mortitlidad geseral. }\end{array}$ & $(31,33)$ & $(26,34,69)$ \\
\hline
\end{tabular}


Situación de salud de la población privada de la libertad en Colombia. Una revisión sistemática de la literatura

Tabla 4. Situación de salud: Problemas o necesidades en salud (continúa)

\begin{tabular}{|c|c|c|c|}
\hline \multicolumn{2}{|c|}{ Situación de salod: problemass o necesidades en salud } & \multirow{2}{*}{$\begin{array}{l}\text { Publicaciones cientificas } \\
(28,33,64,37-39,43)\end{array}$} & \multirow{2}{*}{\begin{tabular}{|l|} 
Literatura gris \\
$(27)$ \\
\end{tabular}} \\
\hline Salud mental & 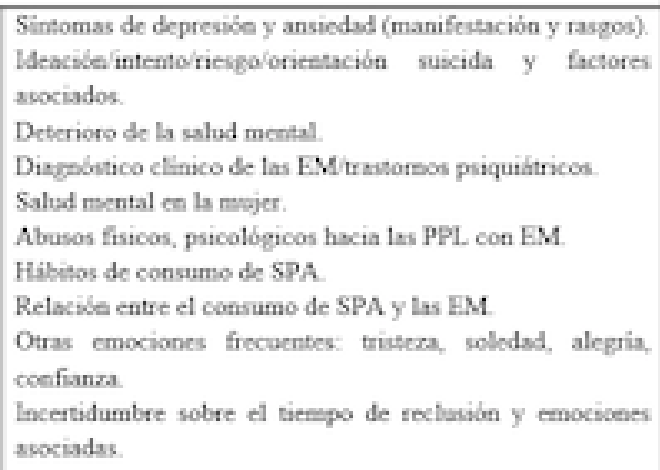 & & \\
\hline $\begin{array}{l}\text { Problemas } \\
\text { nutricionales e } \\
\text { inocuidad de los } \\
\text { alimentos }\end{array}$ & $\begin{array}{l}\text { Deficiescia es castidad y calidad de los alimentos. } \\
\text { Bajo acceso a dietas terapéticas. } \\
\text { Mujeres con baja talla. } \\
\text { lnocuidad de los alimentos }\end{array}$ & $(37,44)$ & (29) \\
\hline \multirow[t]{2}{*}{$\begin{array}{l}\text { Enfermodades } \\
\text { infecciosas }\end{array}$} & $\begin{array}{l}\text { Incidencia y prevalencia de TB latente, palmonar y } \\
\text { extrapulmoent es PPL, comparada coe población general y } \\
\text { factores de riesgo asociados. } \\
\text { Coinfección TB/VTH }\end{array}$ & $(33,54,55)$ & (29) \\
\hline & $\begin{array}{l}\text { Cobertura de tratamiento antituberculoso } \\
\text { Prevalencia de VIH } \\
\text { Prevalencia de Hepatitis B y otras ITS } \\
\text { Conocimiesto do las ITS } \\
\text { Otras: } \\
\text { Deficiencias en el acoeso a los servicios de salud o } \\
\text { medicamentos de las personas con VIH: menos } \\
\text { oportunidades laborales y de integración con otros internos. } \\
\text { Persistencia del estigma y la discriminación asociada con la } \\
\text { infección. } \\
\text { Uticación y convivencia de personas con enfermedades } \\
\text { infecciosas. }\end{array}$ & & \\
\hline $\begin{array}{l}\text { Enfermedades } \\
\text { crónicas no } \\
\text { transenisibles }\end{array}$ & Prevalencia de ECV y diabetes, factores de riesgo asociados. & $(57)$ & \\
\hline
\end{tabular}


Tabla 4. Situación de salud: Problemas o necesidades en salud (continúa)

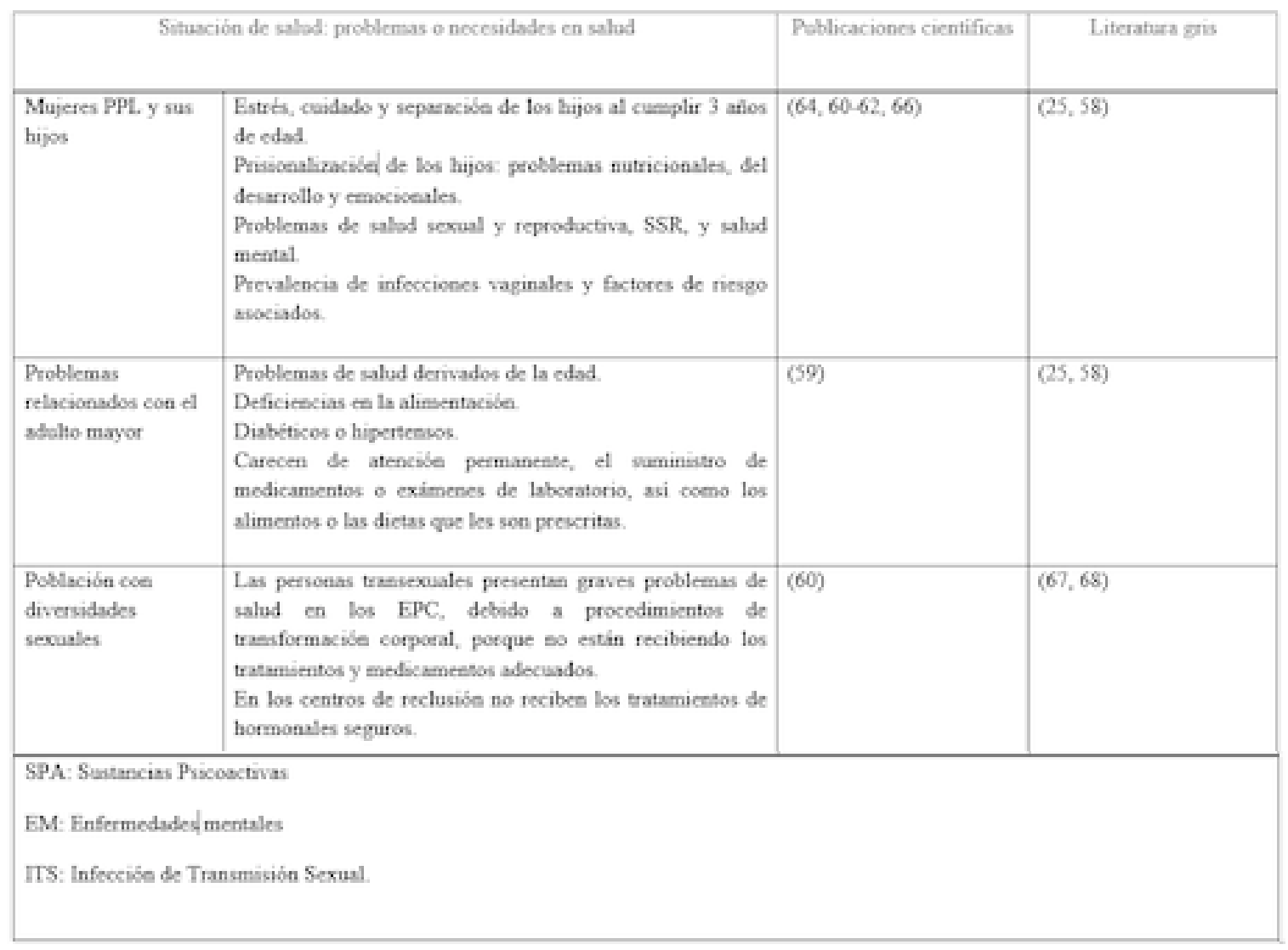

Fuente: elaboración propia.

\section{Discusión}

En Colombia, aunque en los últimos años está aumentando el interés investigativo, existe poca información científica acerca de la situación de salud de las PPL. La mayoría proviene de fuentes oficiales y periodísticas, y son estudios observacionales, con un alto predominio de información sobre las condiciones estructurales y sanitarias, enfermedades infecciosas y problemas de salud mental. El análisis de otras condiciones de salud ha surgido básicamente de los informes que emiten los entes de control del Estado. 
Los estudios e informes revisados sugieren que las PPL atraviesan importantes problemas de infraestructura, hacinamiento y deficientes condiciones de vida, que inciden sobre los múltiples problemas de salud física, mental y ambiental, que según Bello y Parra se configuran como espacios de necropolítica. Según estos autores, la cárcel en Colombia es un lugar "donde los derechos son suspendidos y los cuerpos de las personas presas son expuestos a la enfermedad, el sufrimiento, el abandono y el asesinato" (70).

Los datos muestran persistencia de problemas infecciosos, enfermedades crónicas no transmisibles aún irresueltas como problemas de salud mental y nutricionales, además de importantes problemas y necesidades en salud experimentados por poblaciones especiales. Estas enfermedades representan una carga de morbilidad significativa, que además de afectar a las personas podría estar impactando las finanzas del sistema, bajo el cual se atienden las necesidades de salud de las PPL.

Frente a las enfermedades infecciosas existe una sobreevaluación de la incidencia de la TB, pero muy poca investigación en VIH, y otras ITS, así como otras infecciones por contacto.

De forma similar a las enfermedades infecciosas, los estudios dirigidos a estudiar las enfermedades crónicas no trasmisibles en Colombia también son escasos, solo se encontró uno que calcula la prevalencia de diabetes tipo 2 y ECV, así como sus principales factores de riesgo. No se conocen estudios en hipertensión, asma, diabetes, cardiopatía isquémica, enfermedad pulmonar obstructiva crónica y enfermedad cerebrovascular.

Muy poca literatura aborda los problemas nutricionales en Colombia, y los estudios existentes no son aplicables a todas las PPL, pero los informes de los entes de control han señalado deficiencias en cantidad y calidad de los alimentos. Similar a la situación de Colombia, un estudio realizado con mujeres en Argentina mostró que el principal problema nutricional es el sobrepeso, que afectó al 63,3\%,y el sedentarismo en un 40\% (71).

Con relación a las enfermedades y problemas mentales, aunque no se han caracterizado en investigaciones nacionales, diversos estudios mostraron que los problemas más comunes están relacionados con el consumo de SPA, depresión, ideación o intento de suicidio, otras EM y manifestaciones emocionales. Algunos de estos complejos problemas afectan también al personal de guardia (72). Una alta prevalencia de desórdenes mentales, y en especial de consumo de SPA con baja cobertura terapéutica, también se ha encontrado en otros países como Noruega (73). La OMS ha ilustrado la situación del suicidio y sus causas en los centros de reclusión, y ha propuesto una serie de medidas para mitigar el problema. Según esta entidad, los grupos sociales que más frecuentemente cometen suicidio en prisión son los hombres jóvenes (edades 15-49), las personas ancianas, la población indígena, las personas con enfermedad mental, las personas con abuso de alcohol y/o sustancias, así como quienes han tenido antecedentes de intento de suicidio (74). 
Otras investigaciones encontradas revelaron problemas de salud de las PPL con necesidades especiales como los adultos mayores, las mujeres, los niños que conviven con sus madres en los EPC y las personas con diversidad de género. La creciente prevalencia de enfermedades crónicas y de problemas de salud mental en adultos mayores están relacionadas con las condenas prolongadas (75). Para el caso de las mujeres privadas de la libertad y sus hijos acompañantes, el enfoque ha sido abordado desde un punto de vista biomédico que impide un acercamiento integral a la salud maternoinfantil. Algunos avances en este terreno se han logrado en países como Estados Unidos (65). Por último, la salud de la población LGTBI también se ve desfavorecida por la alta prevalencia de ITS y por los fenómenos culturales de estigma y violencia, tanto física como psicológica, derivados de la ubicación en los EPC, tradicionalmente basada en la genitalidad. Similares hallazgos se encontraron en investigaciones de Estados Unidos (76). En Colombia, solo hasta el 2016 el Inpec incluyó la perspectiva diferencial de esta población mediante la Resolución 6349, pero hay pocos avances en la práctica. El limitado número de estudios referentes a la situación de salud en el país y el alcance local de la mayoría de las investigaciones no permite conocer a fondo la situación objetiva de salud de las PPL. En el mundo, además de los tradicionales problemas de salud, también descritos para Colombia, se abordan otros problemas como las condiciones dermatológicas de las PPL, cáncer de hígado, hepatitis, entre otras $(77,78,79)$. Otros estudios trascienden el momento del confinamiento (80) y evalúan los riesgos y problemas de salud asociados con el retorno a la comunidad y el reingreso a las EPC (81). La escasa literatura acerca de la situación de salud en Colombia es una limitación importante si se quieren proponer políticas o programas de mejoramiento de las condiciones y calidad de vida de las PPL y la garantía de los derechos humanos (82).

Nuestro equipo está consciente de que las investigaciones en los EPC tienen múltiples limitaciones, pero también grandes oportunidades. Las limitaciones son de carácter político, institucional, financieras y prácticas, y están referidas a la falta de prioridad social por parte de los actores del Sistema Nacional de Ciencia y Tecnología, así como de los centros de investigación para promover, financiar y ejecutar investigaciones en esta población, lo que expone a las PPL a una doble exclusión.

Finalmente, el sistema judicial, penitenciario y de salud en Colombia tienen el reto científico, técnico y ético de abordar con rigurosidad y humanidad los problemas de salud que se presentan en los EPC del país, con el propósito de proteger la salud y la vida de las PPL, trabajadores y la comunidad en general con dignidad e integridad.

\section{Agradecimientos}

Al Comité para el Desarrollo de la Investigación, CODI, de la Universidad de Antioquia por la financiación de este estudio. 


\section{Referencias}

1. Quintero-Lyons J, Correa-Solano L. El derecho a la salud a la población carcelaria y penitenciaria en Colombia, en el marco del Estado Social de Derecho. Rev Jur Mario Alario D'Filippo. 2010;2(4):57-76.

2. Colombia. Asamblea General. Resolución 45/111. Principios Básicos para el Tratamiento de los Reclusos Oficina de Naciones Unidas contra la Droga y el Delito. Bogotá: Gobierno de Colombia; 1990.

3. Congreso de Colombia. Ley 65. Por la cual se expide el Código Penitenciario y Carcelario. 40999. Bogotá: Diario Oficial; 1993.

4. Congreso de Colombia. Ley 1709. Por medio de la cual se reforman algunos artículos de la Ley 65 de 1993, de la Ley 599 de 2000, de la Ley 55 de 1985 y se dictan otras disposiciones. 49.039. Bogotá: Diario Oficial; 2014

5. Colombia. Corte Constitucional. Sentencia T-388/13 [Internet]. Bogotá: Corte Constitucional; 2013. Disponible en: http://www.corteconstitucional.gov.co/relatoria/2013/t-388-13.htm

6. Colombia. Corte Constitucional. Sentencia T-277/15 [Internet]. Bogotá: Corte Constitucional; 2015. Disponible en: http://www.corteconstitucional.gov.co/relatoria/2015/t-277-15.htm

7. Higuera LJA, Gómez MAT. Constitución y Cárcel: La judicialización del mundo penitenciario en Colombia. Rev Direito e Práxis. 2019;10:630-660.

8. Artehaga KS, Bedoya MJ. Estándar mínimo de cumplimiento de los derechos constitucionales de las personas privadas de la libertad y su aplicabilidad en el centro penitenciario y carcelario del municipio de Jericó [Trabajo de grado]. [Medellín]: Universidad de San Buenaventura; 2016.

9. Breilh J. La determinación social de la salud como herramienta de transformación hacia una nueva salud pública (salud colectiva). Rev Fac Nac Salud Pública. 2013;31:13-27.

10. Colombia. Corte Constitucional. Sentencia T-762/15 [Internet]. Bogotá: Corte Constitucional; 2015. Disponible en: http://www.corteconstitucional.gov.co/relatoria/2015/t-762-15.htm.

11. Paredes J. Promoción de la salud en el medio penitenciario. En: Colomer C, Álvarez C, editores. Promoción de la salud y cambio social. Barcelona: Masson; 2001. p. 173-189.

12. Colombia. Ministerio de Justicia. Ley 35. Sobre establecimientos de castigo. Bogotá: Diario Oficial; 1914.

13. Mercado-Torres C. De la Antigua Dirección General de prisiones al Inpec, 1914-2014. Cien años de construcción de un sistema carcelario y penitenciario en Colombia. En: Arango-Gonzálesz G, editor. Bogotá: Instituto Nacional Penitenciario y Carcelario; 2004. p. 285.

14. Colombia. Instituto Nacional Penitenciario y Carcelario. Reseña histórica [Internet]. Bogotá: Inpec; 2014. Disponible en: http:/www.inpec.gov.co/portal/page/portal/Inpec/Institucion/ResenaHistorica.

15. Moher D, Liberati A, Tetzlaff J, Altman DG. Preferred reporting items for systematic reviews and metaanalyses: the Prisma statement. PLoS Medicine. 2009;6(7):e1000097. 
16. Toro-Valencia BN. Educación superior en las cárceles colombianas. Acceso a la educación superior en las Instituciones carcelarias y penitenciarias de Colombia. Cali: Instituto Internacional para la Educación Superior en América Latina y el Caribe; 2005.

17. Colombia. Consejo Nacional de Política Económica y Social/Departamento Nacional de Planeación. Conpes 3828. Política Penitenciaria y Carcelaria en Colombia Bogotá: Consejo Superior de Política Criminal ; 2015.

18. Marcos F, Morris T-B, Yrigoyen R. Misión Internacional Derechos Humanos y Situación Carcelaria. Centros de Reclusión en Colombia: un Estado de Cosas Inconstitucional y de Flagrante Violación de Derechos Humanos. Bogotá: Naciones Unudas; 2001.

19. Colombia. Defensoría del Pueblo. Decimonoveno Informe del Defensor del Pueblo al Congreso de la República de Colombia. Bogotá: Defensoría del Pueblo; 2012. p. 640.

20. Colombia. Consejo Nacional de Política Económica y Social. Conpes 2797. Política Penitenciaria y Carcelaria [Internet]. Bogotá: Departamento Nacional de Planeación; 1994. Disponible en: https://c olaboracion.dnp.gov.co/CDT/CONPES/Econ\%C3\%B3micos/2797.pdf

21. Colombia. Defensoría del Pueblo. Informe del Defensor del Pueblo de Colombia al Congreso de la República. Segunda Parte. Bogotá: Defensoría del pueblo; 2016.

22. Institute for Criminal Policy Research, Birkbeck University of London. Highest to Lowest - Prison Population Total [Internet]. Disponible en: http://www.prisonstudies.org/highest-to-lowest/prison-p opulation-total?field_region_taxonomy_tid=All.

23. Bargent J. El reflejo de Colombia: guerra y narcotráfico en el sistema penitenciario [Internet]. Washington: Centro de Investigación de Crimen Organizado; 2017.Disponible en: http://es.insightcr ime.org/investigaciones/reflejo-colombia-guerra-narcotrafico-sistema-penitenciario.

24. British Broadcasting Corporation. Crisis tras las rejas en Latinoamérica Reino Unido. BBC Mundo; 2005. Disponible en: http://news.bbc.co.uk/hi/spanish/specials/2005/carceles/newsid_4331000/4331 358.stm.

25. Instituto Nacional Penitenciario y Carcelario. Población Intramural. 2017. Disponible en: http://201.217.206.18:8080/jasperserver-pro/flow.html?_flowId=dashboardRuntimeFlow\&dash boardResource=/public/DEV/dashboards/Dash_Poblacion_Intramural\&j_username=inpec_user\&j password $=$ inpec

26. Colombia. Instituto Nacional Penitenciario y Carcelario. Estadísticas 2017. Bogotá: Ministerio de Justicia; 2017.

27. Quintero R, Báez C. El País Detrás de las Rejas. El Tiempo. 2018.

28. Sánchez-Ruiz DE. Situación de salud en un centro penitenciario - Colombia, 2013 - 2014. Medellín: Universidad de Antioquia; 2015.

29. Oficina en Colombia del Alto Comisionado de las Naciones Unidas para los Derechos Humanos. Desde la Prisión. Realidades de las Cárceles en Colombia. Bogotá: Naciones Unidas, Alto Comisionado; 2006. 
30. Colombia: Defensoría del Pueblo. Situación del Servicio de Salud en las Carceles de Colombia. Bogotá: Defensoría del Pueblo; 2003. p. 43.

31. Alvárez-Correa M, Cadena G. Del timbo al tambo. Salud mental en el sistema carcelario colombiano y política pública. Rev Salud Bosque. 2019;9(1):63-83.

32. Colombia. Defensoría del Pueblo. 23 Informe del Defensor del Pueblo al Congreso de la República de Colombia. Segunda Parte. Bogotá: Defensoría del Pueblo; 2015.

33. Riaño A, Chamorro-Mora S. Morbilidad en un centro penitenciario de Colombia. Rev Fac Cienc Salud Univ Cauca. 2019;21(1):16-22.

34. Colombia. Defensoría del Pueblo. Vigésimo Informe del Defensor del Pueblo al Congreso de la República. Informe Ejecutivo Porque los derechos humanos están presentes en todos los momentos de la vida. Bogotá: Defensoría del Pueblo; 2013.

35. Colombia. Defensoría del Pueblo. Informe atención en salud mental a población privada de la libertad [Internet]. Bogotá: Defensoría del Pueblo; 2015. Disponible en: http://www.defensoria.gov.co/publ ic/pdf/01/Atencion-en-salud-mental-2015.pdf.

36. Maldonado-Fuentes F. Prevalencia de patologías de salud mental en la población adolescente privada de libertad: experiencias nacionales y comparadas. Ius et Praxis. 2013;19(1):329-362.

37. Jaramillo CP, Benjumea MV. Diagnóstico situacional de las internas del reclusorio de mujeres de Manizales. Hacia Promoc Salud. 2007;12(1):1-15.

38. Uribe-Rodríguez AF, Martínez-Rodríguez M, López-Romero KA. Depresión y ansiedad estado/rasgo en internos adscritos al "Programa de Inducción al Tratamiento Penitenciario" en Bucaramanga, Colombia. Revista Criminalidad. 2012;54(2):47-60.

39. Pérez J, Gómez I, Landazabal M, Morales S, Sánchez-Mendoza V, Páez D. Riesgo de suicidio en prisión y factores asociados: un estudio exploratorio en cinco centros penales de Bogotá. Rev Colomb Psicol. 2002;11(1): 99-114.

40. Jaramillo-Gutiérrez MR, Silva-Vallejo C, Rojas-Arango BP, Medina-Pérez ÓA. Ideación suicida y factores asociados en internos de un establecimiento penitenciario de Antioquia (Colombia). Rev Colomb Psiquiatr. 2015;44(2):100-105.

41. Mojica CA, Sáenz DA, Rey-Anacona CA. Riesgo suicida, desesperanza y depresión en internos de un establecimiento carcelario colombiano. Revista Colomb Psiquiatr. 2009;38(4):681-692.

42. Serrano-Tárraga MD. Derecho a la salud de los internos en centros penitenciarios y sanidad penitenciaria (II). Rev Derecho Uned. 2010;7:525-260.

43. Ruiz JI. Clima emocional y sobreocupación en prisión: una evaluación mediante informantes clave. Suma Psicológica. 2006;13(2):159-172.

44. Bejarano-Roncancio J, Celedón-Dangond C, Socha-Gracia L. Alimentación penitenciaria: entre higiene y derechos. Rev. Fac. Med. 2015;63(3):527.

45. Almeida-Rueda L, Giraldo-Pineda A, Forero-Pulido C. "Encierro sobre el encierro" aislamiento por tuberculosis. Centro penitenciario Medellín Colombia. Rev Univ Ind Santander Salud. 2016;48(1):61-69. 
46. Colombia. Defensoría del Pueblo. Decimoctavo Informe del Defensor del Pueblo al Congreso de la República. Bogotá: Defensoría del Pueblo; 2011.

47. Roldán J, González L. Situación de los Derechos Humanos en las cárceles del área Metropolitana. Diagnóstico sobre los derechos humanos en las cárceles: Bellavista, La Estrella, Itagüí. Reclusión de Mujeres Buen Pastor y San Quintín- Bello. Medellín: Cáritas Arquidiosesana Medellín/Pastoral Social; 2005.

48. Rueda ZV, Arroyave L, Marin D, Lopez L, Keynan Y, Giraldo MR, et al. High prevalence and risk factors associated with latent tuberculous infection in two Colombian prisons. The international journal of tuberculosis and lung disease. Int J Tuberc Lung Dis : the official journal of the International Union against Tuberculosis and Lung Disease. 2014;18(10):1166-1171.

49. Rueda ZV, López L, Vélez LA, Marín D, Giraldo MR, Pulido H, et al. High Incidence of Tuberculosis, Low Sensitivity of Current Diagnostic Scheme and Prolonged Culture Positivity in Four Colombian Prisons. A Cohort Study. PLoS ONE. 2013;8(11):e80592.

50. Gómez IT, Llerena CR, Zabaleta AP. Tuberculosis y tuberculosis farmacorresistente en personas privadas de la libertad. Colombia, 2010-2012. Rev Salud Pública. 2015;17(1):97-105.

51. Arenas N, Torres E, Durango C, Cuervo L, Coronado S, Gómez A. Búsqueda Activa de Individuos con Tuberculosis Pulmonar y Extrapulmonar en Calarcá-Quindío, Colombia-2005. Rev Salud Pública. 2008;10(2):279-289.

52. Gaviria-García G, Lastre-Amell G, Trejos-Herrera A. Prevalencia del VIH y conductas de riesgo en internos de un centro carcelario del distrito de Barranquilla (Colombia). Rev Salud Uninorte. 2015;31(1):25-35.

53. Calonje ET, Norella-Córdoba D. Virus de inmunodeficiencia humana: un desafío en las cárceles en Colombia. Rev Salud Bosque. 2018;8(2):49-51.

54. De la Hoz F, Velandia-Gonzalez MP, Martinez M, Varona M, Rojas C, Gómez H, et al. Estudio de un brote de hepatitis B en la cárcel de una ciudad intermedia colombiana 1992-1993. Infectio. 1995;1(1):6-11.

55. Castañeda-Porras O, Daza-Camelo L, Díaz-Criollo S, Moreno-Cháves T, Segura-Durán O. Hepatitis B en el establecimiento penitenciario de La Dorada, Caldas, Colombia, 2009. MedUnab. 2011;14(1):32-59.

56. Colombia. Defensoría del Pueblo. Defensoría: la declaración de emergencia carcelaria es insuficiente [Internet]. Noticias RCN. 2017. Disponible en: https://noticias.canalrcn.com/nacional-pais/defensor ia-declaracion-emergencia-carcelaria-insuficiente

57. Ochoa-Orozco SA, Moreno-Gutiérrez PA, Echeverri-Cataño LF, Orozco-Escobar A, MondragónCardona Á, Villegas-Rojas S. Riesgo cardiovascular y de diabetes en población carcelaria de Pereira, Colombia, 2010. Rev Méd Risaralda. 2012;18(2):129-133.

58. Abaunza-Forero CI, Bustos-Benítez P, Enríquez-Wilches K, Mendoza-Molina M, Padilla-Muñoz A, Paredes-Álvarez G. Adulto mayor: prisión y sociedad. En: Universidad Externado de Colombia, editor. Política Criminal y Libertad. Bogotá: Universidad Externado de Colombia; 2014. p. 271-306. 
59. Abaunza-Forero CI, Mendoza-Molina MA, Bustos-Benítez P, Paredes-Álvarez G, Enriquez-Wilches KV, Padilla-Muñoz AC. Adultos mayores privados de la libertad en Colombia. Bogotá: Universidad del Rosario/Instituto Rosarista de Acción Social, Seres; 2014.

60. del Pozo Serrano FJ, Martínez-Idárraga JA. Retos del tratamiento penitenciario en Colombia: enfoque y acción diferencial de género desde la perspectiva internacional. Revista Criminalidad. 2015;57(1):9-25.

61. Briceño-Donn M. Mujeres y prisión en Colombia: Análisis desde una perspectiva de Derechos Humanos y género. Bogotá: Corte Interamericana de Derechos Humanos; 2006.

62. Ariza LJ, Iturralde M. Una perspectiva general sobre mujeres y prisiones en América Latina y Colombia. Revista Derecho Público. 2015;32(1):4-25.

63. López-Barbosa N, Castro-Jiménez MÁ, Gamboa-Delgado EM, Vera-Cala LM. Prevalencia y determinantes de las infecciones vaginales en las mujeres recluidas en una cárcel colombiana. Rev Chil Obstet Ginecol. 2009;74(2):77-82.

64. Aristizábal ET, Ríos-García AL, del Pozo-Serrano FJ. Salud Mental, género, educación social en mujeres reclusas del Centro de Rehabilitación Femenino El Buen Pastor de Barranquilla (Colombia) (2015-2016). Salud Uninorte. 2016;32(2):256-257.

65. Shaw J, Downe S, Kingdon C. Systematic mixed-methods review of interventions, outcomes and experiences for imprisoned pregnant women. J Adv Nurs. 2015;71(7):1451-1463.

66. Colombia. Defensoría del Pueblo. Los derechos humanos de la mujer privada de la libertad en Colombia. Bogotá: Defensoría del Pueblo; 2011.

67. Colombia: Comisión de seguimiento de la sentencia. Segundo informe de la comisión de seguimiento de la sociedad civil a la sentencia T-388 de 2013 de la Corte Constitucional. Unaula. 2017;3(5):81-115

68. Parra-Pérez LC. La verdad de la comunidad LGTBI en las cárceles colombianas. Bogotá: Universidad Militar Nueva Granada; 2015.

69. Personería de Medellín. Informe sobre la situación de los derechos humanos en la ciudad de Medellín, 2015. Medellín: Personería de Medellín; 2015.

70. Gallego G, Ramírez J. Cárceles de la muerte: necropolítica y sistema carcelario en Colombia. Universitas Humanística. 2016;82(1):365-391.

71. Nessier MC, Gerlero SS. "El estado nutricional tras las rejas", estudio descriptivo del estado nutricional de un grupo de mujeres privadas de libertad en una unidad penal de la Ciudad de Santa Fe (Argentina). Rev Esp Nutr Comunitaria. 2012;18(2):91-97.

72. Rodríguez-Amaya RM. Burnout Syndrome in Prison Guards, Bucaramanga, Colombia, 2013. Rev Colomb Psiquiatr. 2014;43(3):146-153.

73. Yi Y, Turney K, Wildeman C. Mental Health Among Jail and Prison Inmates. Am J Mens Health. 2017;11(4):900-909.

74. Organización Mundial de la Salud/Asociación Internacional para la Prevención del Suicidio. Prevención del suicidio en cárceles y prisiones. Ginebra: OMS; 2007. 
75. Forsyth K, Archer-Power L, Senior J, Meacock R, Webb R, Emsley R, et al. Health Services and Delivery Research. The effectiveness of the Older prisoner Health and Social Care Assessment and Plan (OHSCAP): a randomised controlled trial. Southampton(UK). NIHR Journals Library; 2017 dic.

76. White-Hughto J, Clark-Kirsty A, Altice-Frederick L, Reisner-Sari L, Kershaw-Trace S, Pachankis JE. Creating, reinforcing, and resisting the gender binary: a qualitative study of transgender women's healthcare experiences in sex-segregated jails and prisons. Int. J. Prison. Health. 2018;14(2):69-88.

77. Coury C, Kelly B. Prison dermatology: experience in the Texas Department of Criminal Justice dermatology clinic. J. Correct. Health Care. 2012;18(4):302-308.

78. Baillargeon J, Snyder N, Soloway RD, Paar D, Baillargeon G, Spaulding AC, et al. Hepatocellular Carcinoma Prevalence and Mortality in a Male State Prison Population. Public Health Reports. 2009;124(1):120-126.

79. Pontali E, Ferrari F. Prevalence of Hepatitis B virus and/or Hepatitis C virus co-infections in prisoners infected with the Human Immunodeficiency Virus. Int J Prison Health. 2008;4(2):77-82.

80. Spaulding AC, Seals RM, McCallum VA, Perez SD, Brzozowski AK, Steenland NK. Prisoner survival inside and outside of the institution: implications for health-care planning. Am Journal of Epidemiology. 2011;173(5):479-487.

81. Patterson GT. Prisoner reentry: a public health or public safety issue for social work practice? Soc Work Public Health. 2013;28(2):129-141.

82. Coyle A. Health care and the prisoner: a human rights perspective. Clin. Forensic Med. 1997;4(4):181-184.

\section{Notas}

Artículo de revisión

1 Los alimentos sin control corresponden a donaciones, elaboración de bebidas artesanales alcohólicas internamente, y alimentos ingresados por los familiares y las visitas. 